\title{
An approach to model forgetting
}

\author{
Jennifer Haase ${ }^{1[000-0001-8450-7252]}$ and Christof Thim ${ }^{1[0000-0003-0016-8180]}$ \\ University of Potsdam, 14482 Potsdam, Germany, [jhaase, cthim] @lswi.de, https://lswi.de
}

\begin{abstract}
This paper aims to investigate the possibility to include aspects of forgetting into business process modeling. To date, there is no possibility to model forgotten or to-beforgotten elements beyond the mere deletion. On a first attempt, we focus on the individual level and model knowledge transformation within a single person. Using the Knowledge Model Description Language, we propose ways to include different forms of forgetting into the realm of modeling notations. Using data from an experimental setting within an assembly line production environment, the feasibility of those new modeling notations is tested. So far, the applicability of modeling features for forgetting on the individual level is mostly restricted to a research context. However, clear requirements to transfer the notations onto the team- and organizational level are set out.
\end{abstract}

Keywords: business process modeling $\cdot$ forms of forgetting $\cdot$ KMDL $\cdot$ modeling tools

\section{Introduction}

Most task in the work context require that previously acquired knowledge has to be put into action. Companies spend large amounts of money on training for their employees and to accumulate more and more knowledge about their business objects, their tools and their social interaction. However, when thinking about the transformation of businesses, learning alone might not be enough. Employees might still possess knowledge about former means and ends of their tasks which are not considered feasible anymore. This unwanted knowledge can be overpowered by newly learned content, yet as psychological research shows, there are methods to increase the chance of successfully putting aside obsolete information [1].

Forgetting is an essential and most functional feature of the human brain, although it is much more often perceived as a failure and weakness of memory [25]. This contempt of the adaptive nature of forgetting might be due to the fact that when forgetting happens, we most often just do not realize it. If we loose something unimportant, how would we know? Plus, given the assumption that memory items serve the purpose of dealing with the present and anticipating the future, all too detailed knowledge about the past does not help with finding patterns and drawing conclusions [30]. Taking the human brain as an analogy to a companies knowledge base, it is easy to assume that also companies could profit from forgetting mechanisms [33]. Whenever change is needed in order to adapt to new working conditions, the process of adaption asks for the acquisition of new knowledge. Usually the decision what to acquire is derived from assumed future needs. However, the starting point of the change, the current knowledge base, is much less often considered, although previous knowledge partly determines what can be learned easily [15]. When prior knowledge exists, additional knowledge can much easier be added to that, compared to a new topic being learned from scratch. On the contrary, the knowledge which became obsolete and thus needs to be substituted is much harder to erase, as this outdated knowledge can interfere with the new to acquire one, especially the more the fact is over-learned and routinized [1,6]. Due to these phenomena it matters whether change needs learning of new information or the substitution of old information. When a production process is altered, for example to include new technology, the workaround changes in a way that some parts become obsolete and some are added to the original one. This means workers learn and substitute information, which requires different cognitive capacities and attentiveness, respectively. On the individual level the desired transformation can be described by means of the concept of intentional forgetting [19].

Forgetting is not limited to the individual level. Changes in the organizational context often touch entire business processes. Forgetting therefore also occurs on the process level, where tasks, teams, work environment and interaction patterns are changed. This also includes the discharge of obsolete processes, knowledge and rules [8]. Organizational forgetting than interacts with individual forgetting, which can enforce or hamper the adaption process. 
Organizational change is usually guided by management measures. One way to describe the transformation is to use formalized business process models. They depict the tasks, resources and relationships in their initial state and provide a specification of the desired state. In order to aid the organisational transformation, individual forgetting can be directed. To identify potential knowledge resources and settings that hamper this process, business process models need to encompass forgetting. Therefore the central research question in this paper is: How can different forms of forgetting be modelled in business processes?

To answer this question, we first explore which organizational elements are involved in forgetting. To this end, the level on which forgetting occurs and the necessary requirements are defined. This is substantiated by different types of forgetting from psychological theory. These findings are conflated into an approach to capture forgetting in business processes. We demonstrate the feasibility of our modelling patterns by deploying the approach to process changes in an experimental setting.

\section{Forgetting within business processes}

Business process modelling is an approach to cope with the complexity of business processes. In most cases those models depict the resource allocation and the resource flow transforming inputs to outputs within an organization. Depending on the perspective taken, they can concentrate on different business aspects. As most companies already deploy some sort of process management, tasks and resources are already mapped out inside the company [24].

A precise modeling of business processes helps with the understanding of complex intertwined processes within a company, and thus can most perfectly be used to also map change processes. The old process can be mirrored, presenting the status quo, whereas the new process then represents the vision, the goal which is aimed to achieve. Comparing those can shed light on those parts which will become obsolete and uncover those gaps where new knowledge needs to be acquired. Thus, also the analysis of the elements which need to be forgotten can be part of the change-strategy.

However, the most prominent modelling notations (e.g. BPMN, eEPC or UML) only concentrate on tangible resources such as machines, personal, information, their association and their transformation flows. Here forgetting can only be observed by a change in the association pattern or if flows of tasks are altered. They disregard the individual level. Yet, most activities within the economy are knowledge intensive, meaning that their inputs and outputs are diverse and uncertain [7]. Therefore a business process is knowledge intensive if value can only be created by the fulfillment of the knowledge requirements by the process participant [11]. The above mentioned modeling notations do not have the ability to describe the use and transformation of knowledge within business processes. Only a few extensions provide the means to capture these intangible resources and processes. Sigmanek and Lantow [31] provide an overview over these modeling notations. Modeling elements can be used to provide a precise and realistic representation of the knowledge development of individuals, groups and organizational units. This representation can be used to identify individual knowledge gaps but also knowledge elements which are unneeded. We argue that forgetting is a dynamic knowledge transformation process similar to learning, which can be captured by related concepts.

So in order to define the specifics of an approach to model forgetting in business processes, the requirements for such a notation need to be deducted from modeling theory as well as from psychological findings regarding forgetting.

\subsection{Requirements for modeling forgetting}

In accordance with the Guidelines of Modelling [3] the approach to model forgetting has to be relevant, correct and economically efficient. Furthermore models should be clear, comparable and systematically designed. We therefore derive the requirements from the first three aspects of the modelling guidelines. The latter three are used in the development process as validation points.

Relevance as the first criteria requires the selection of a specific domain or purpose, which the model serves. The purpose of our models and hence the developed modeling approach is to aid the change in business processes by facilitating intentional forgetting. We therefore need to consider the relevant elements of forgetting and their dynamic association. The latter can be defined by different types identified in section 2.3. The elements of forgetting in business processes however can be derived from a definition of forgetting and a thorough analysis of the entities involved. 
We have to distinguish two different levels on which forgetting can occur. On the individual level forgetting can be defined as the failure or inability of an individual to retrieve previously acquired memory items [27]. Forgetting is therefore a psychological process which describes changes in the accessibility of the individual's knowledge base.

This is also true on the organizational level. However, the organizational knowledge base differs. Where the individual's knowledge can be described neurologically, the organization's knowledge is distinctly social, containing it's members individual knowledge as well as the rules, norms and values governing the interaction [19].

In order to model forgetting, the individual level is a good point of reference which can be later extended to include interaction and governance. The focus on the individual level influences the requirements for the modeling notation. Most notations use a generic approach to model business processes. They operate on a schema level. Since knowledge and forgetting are highly individual and time dependent, this is not sufficient for our modeling purposes. Forgetting occurs in specific process instances with specific resources and tasks. The first requirement is therefore: A notation to model forgetting should be able to capture knowledge flows and transformations on an instance level.

Closely related to the instance-level-requirement is the necessity to capture the relationship between knowledge and its bearers. Some modeling notations only semantically describe knowledge as a relation of different knowledge items or their association to different tasks. This general description does not capture the personal task execution which puts knowledge into action. No conclusions about individual forgetting can be drawn from this modeling perspective. Furthermore the semantical description of knowledge cannot be used to aid the change of business processes. Since most measures of change management are either related to the allocation of employees to tasks or to the skill set of these employees, we propose the second requirement, which accounts for the central role of the employee: sufficient modeling notation needs to provide means of describing concrete knowledge-person-task relations.

In order to capture the dynamics in knowledge development a mere semantic indication of which knowledge objects exist and their relations is not enough. Each knowledge object can undergo a shift within itself. Concrete knowledge from experience can become more abstract with some theoretical foundation or a number of observations. Knowledge acquired from books on the other hand can be complemented with experiences from concrete interaction with the environment. Also knowledge can be tacit or explicit, depending on the degree to which its content can be articulated. These shifts and dynamics have to be captured in order to distinguish different levels and gradual development in forgetting. Where shallow structures of explicit knowledge can be forgotten more easily, forgetting will be much more complicated for knowledge in deep structures which is intertwined with other knowledge elements. Knowledge therefore has to have some kind of quantification, possibly in multiple dimensions. Our third requirement is therefore: Knowledge objects should possess a sufficiently dense description. They should not only name the content, but offer enriched information about the level of insight and skill which an actor possesses.

In summary, the following three requirements are used to ensure the relevance of the modeling approach. A sufficient modeling notation should: 1. be able to capture knowledge flows and transformations on an instance level (instance). 2. provide means of describing concrete knowledgeperson-task relations (person). 3. provide a dense description of knowledge objects exceeding mere semantic description (operationalization).

\subsection{Evaluating existing modeling approaches}

To select a suitable notation of modeling forgetting in business processes one can either develop a new modeling language from scratch or extent a suitable existing approach. With regard to the correctness and efficiency goal of modeling [3] the selection of an appropriate existing notation is to be preferred. First of all, the foundation of an existing meta-model can ensure syntactic correctness. Also semantic correctness can better be achieved with regard to an existing notation and procedure model from a related domain.

As it was brought forward in section 2.1, forgetting is strongly related to knowledge dynamics and knowledge management. There is a variety of possible approaches to model knowledge intensive processes $[9,10]$ however most approaches are bound to a specific domain. The following approaches originally brought forward by Sigmanek and Lantow [31] will be described in short and then evaluated against the requirements laid out in section 2.1: 
- Declarative Configurable (DeCo) [28]

- Business Process Oriented Knowledge Management (GPO-WM) [17]

- Knowledge Intensive Process Notation (KIPN) [22]

- Knowledge Modeling and Description Language (KMDL) [11]

- Knowledge Process Redesign (KPR) [2]

- PROMOTE [18]

$D e C o[28]$ has the primary objective to aid process execution for tasks which are only partially structured. It deploys declarative modeling, model verification and variability modeling techniques. The basic assumption is, that in knowledge intensive processes the flow of tasks cannot be predefined and has to be generated on the fly. DeCo-models are therefore maximally configurable and have no fixed constrains. In the modeling process pre-defined (structured) process parts are modeled separately from knowledge intensive processes which are defined during deployment of a process model as an instance. DeCo is therefore able to model at the instance level. However due to the strong process and task automation focus, DeCo does not provide a link between knowledge and person, although it is an adaption of the BPMN-standard with optional objects. Furthermore is doesn't provide any possibility to describe the knowledge elements in the processes more thoroughly. GPO-WM [17] has been developed in order to capture the basic tasks of knowledge management: generating, storing, transferring and applying knowledge. It is more a procedural model than an actual modeling notation. It does not provide any predefined attributes and therefore produces vague and incomparable models. Also there are no means to analytically assess the models. GPO-WM only provides guiding questions and best practices to aid the identified knowledge management tasks. It therefore does not fulfill any of the requirements.

$K I P N[22]$ is based on the findings that standard modeling notations such as BPMN or EPC do not provide the means to address the requirements for knowledge intensive process [26], especially they do not capture poorly structured processes, knowledge transfer, the relationship between different processes as well as the short lifespan of knowledge. KIPN therefore develops an ontological approach with five different diagrams. It captures business rules, decisions and control flows on a process level. On the individual level it uses intention diagrams to depict individual motivations and motivation chains for specific tasks. The interaction between different employees is displayed in socialization diagrams, which address the flow of communication. The detailed modeling approach fulfills the instance and knowledge-person requirement. However, knowledge objects are only named not operationalized. Hence KIPN fails this requirement.

$K M D L$ [11] provides different means to identify and model knowledge intensive processes. Its main goal is to capture the knowledge transitions laid out by Nonaka and Takeushi [23]. It relates tasks from the control flow (process view) to knowledge transitions like socialization, externalization, internalization and combination in the activity view. Each activity constitutes a process instance in which knowledge which is bound to a specific person is altered or transferred. In a further development knowledge objects are attributed with quantifiable measures for their explicability, generality, experience-boundness and level of insight [12]. It therefore fulfills all of our requirements.

$K P R$ [2] focuses on the tasks of knowledge access and sharing. Its main objective is the configuration of knowledge management systems and the integration of these systems into the process flows. In order to achieve this, it is integrated into the ARIS-framework and uses EPC to capture the process and knowledge flows. EPC is extended by knowledge structograms which identify knowledge carriers, categories and needs. The combination of the process model and the knowledge structogram enables KPR to identify unmet knowledge needs, monopolies and inadequate employee profiles. Respective measures can be taken to ensure the efficient access to and sharing of knowledge by means of technological knowledge management systems. Since it is operating on an abstract process level, it does not consider specific instances. However it captures persons and their knowledge and allows to assess the value of this knowledge within the business process.

PROMOTE [18] also focuses on knowledge generation and search within business processes. Its aim is to integrate these tasks into a software solution. The notation is used to distinguish different knowledge flows from external actors into the company, from the company to the environment and within the company. The resultant software solution is designed to aid these flows and make them more transparent and efficient. The PROMOTE-approach is technically oriented, but considers process instances in its modeling. Knowledge is only viewed abstractly, it is therefore not operationalized and not linked to its carriers. 


\begin{tabular}{lccc}
\hline Approach & Instance & Person & Quantification \\
\hline DeCo & yes & no & no \\
GPO-WM & no & no & no \\
KIPN & yes & yes & no \\
KMDL & yes & yes & yes \\
KPR & no & yes & yes \\
PROMOTE & yes & no & no \\
\multicolumn{2}{r}{ Table 1. Fit between approaches and requirements }
\end{tabular}

Evaluating the six modeling approaches generates the results in Table 1. We find that KMDL $[11,12]$ is best suited to fulfill the requirements defined in section 2.1. We therefore use its elements and notations and adapt and extend their usage. We concentrate on the activity view where concrete knowledge transitions are modeled. As we have defined the purpose and the means of modeling forgetting, we need to further differentiate different types of forgetting before we can generate an approach to capture forgetting in business processes.

\section{$2.3 \quad$ Types of forgetting}

So far, forgetting was portrayed as a unitary concept, which is based on the definition that forgetting describes the inability to retrieve once known information. However, there are several ways how this inability is caused. The most common forms are trace decay, interference, cue-dependent forgetting and intentional forgetting, which will all be explained shortly.

Trace decay is the simplest form of forgetting, where the association with a knowledge content weakens over time due to not-activation. This alleviated association drops under a threshold where a memory content can then not be remembered anymore [21]. The result is the appearance of memory loss.

Another form of forgetting is interference, which happens when associations to old memory content weakens due to the acquisition of a new but similar memory content (this is called retroactive interference. Proactive explains the phenomenon that new memory content cannot be learned due to old and similar memory content, but since this focuses more on learning than forgetting, we only consider retroactive interference) [6]. The result of retroactive inference is again a weakened association which can lead to the inability to recall the memory, whereas the new memory content was learned and thus can be remembered. In literature, it was long debated whether true decay happens, or if all memory loss is rather due to interference of some sort. Newer studies show, that both mechanisms take place, but it depends on the kind of memory how it might be forgotten [16, 29].

Another way how memory content can become inaccessible is due to its cue relation. In cuedependent forgetting, a memory content gets associated with a cue during the encoding process. This cue can be of any sort of sensory input [19]. If this cue is not present, the memory content will most probably not be recalled. Yet, there is a chance though, that the original memory content became over-learned over time, so that it can be remembered, even without the cue (called generalization) [32].

A special form of cue-dependent forgetting takes place when two different memory items get associated to the same cue (called cue-overload). If the cue is present, four different scenarios can happen: the new memory content could get retrieved at the cost of the primary memory content. Second, only the first memory content could be retrieved, if this association is strong enough to compete with the newly learned, and thus still weak association of the second memory content. Third, both memory items could be retrieved, which might result is a confusion-like state. Forth and last, the cue can become obsolete and thus no memory item gets retrieved.

The former types of forgetting are all of mere passive, thus accidental form. However, if a person actively wants to forget, we speak of intentional forgetting. Here, the instruction to forget, coming from outside or within a person, leads to a suppression of associated or retrieved knowledge, when the memory item became obsolete [19]. It is actually quite difficult to predict what happens to the information, because the suppression of knowledge does not automatically lead to decay, although it is possible. Some evidence even points to the opposite effect, that when information purposefully 
shall be forgotten, it gets better remembered, at least at a subconscious level [5]. We use the concept so far in a pragmatic way, by defining it as the purposeful not-usage of information, no matter the actual cognitive presentation.

\section{An Approach to Model Forgetting}

The only way to model forgetting with current approaches is to delete obsolete objects. By comparing two models of the same process, the deleted elements are identified as forgotten. This approach does not allow for the detection of the forgetting process and its impact on related elements. Coming from classical modeling theories, a conflict arises based on the premise of parsimony in modeling [24]. In order to manipulate forgetting it has to become visible and detectable. It thus takes a concept shift, so forgotten elements are not perceived as simply gone, but as still useful elements. There are different levels within an organization where forgetting can be of importance and thus should be detected in order to use it within business process modeling: individual, team and organizational [4].

Our modeling approach is based on the elements provided by KMDL: knowledge object, person, conversions, information object as a cue and requirements (compare Table 2, for detailed explanation see [13]). In KMDL information objects are part of the knowledge flow, e.g. as an output from an externalization. The flow is depicted with a directed edge. Since we use information objects as cues in the forgetting process, they need to be modeled differently. A cue can activate the retrieval of a knowledge object. It therefore governs the association between a knowledge object and its carrier. We therefore use an un-directed edge associated with the connection between person and knowledge object to depict this relationship.

\begin{tabular}{lll}
\hline Objective & Representation & Description \\
\hline Knowledge object & square-like vertice & illustrates knowledge, competences, skills, \\
& experiences, attitudes and behavior of a person \\
Person & oval-like vertice & $\begin{array}{l}\text { represents a knowledge keeper, like a real person } \\
\text { within the organization }\end{array}$ \\
Information object & arrow-like vertice & represents explicit knowledge, which can exist \\
& & outside of a person \\
Requirement & square-like vertice with & symbolizes what is needed in order to fulfill a \\
& two concave lines & conversion, like a tool or certain soft-skills \\
Conversion & oval-shaped vertice & an activity within a knowledge flow, according \\
& & to the types of knowledge conversions laid out \\
Knowledge flow & an undirected edge & by Nonaka and Takeushi [23] \\
& & object and its carrier
\end{tabular}

Table 2. Elements of KMDL

So far, KMDL represent a static process, whether the status quo or the vision of the goal process. However, per definition, forgetting can only be addressed with a focus on knowledge dynamics. We solve this issue by introducing several time points, focusing on changes over time. We can thereby model forgetting as multi-step processes reaching from $t_{0}$ to $t_{n}$. Further, since learning is a precondition for forgetting, we start the modeling with an already learned and thus associated knowledge object.

As explained above, decay describes the simplest form of forgetting, where the once learned association with a knowledge object lessens over time, until it cannot be retrieved anymore (see Fig. 1). The decaying association is modeled using a dashed instead of a solid edge and represents the inability to recall the knowledge. This representation is a newly added feature of KMDL and 
represents the elementary type of forgetting as a foundation for all other types of forgetting. We specify the representation of forgetting as a decayed association (dashed line) between a person and an knowledge object.

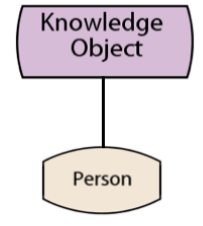

$t_{0}$

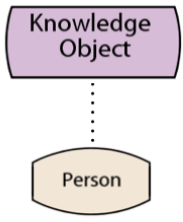

$t_{1}$

Fig. 1. Modeling memory content loss due to trace decay.

Interference is represented by a second knowledge object being learned, which has a negative impact on the association from the first knowledge object, symbolized by a minus added to the link of the first knowledge object. This leads at the end to a new association with the second knowledge object, at the cost of the first (see Fig. 2). This feature is also a new one added to KMDL.

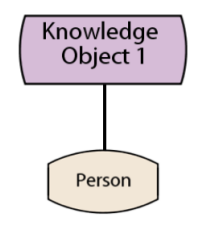

$t_{0}$

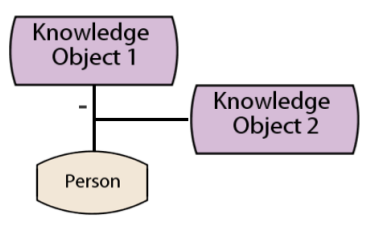

$t_{1}$

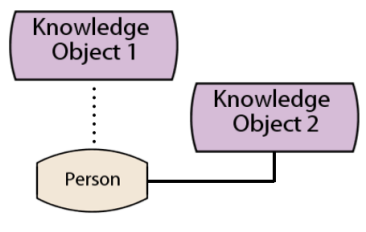

$t_{2}$

Fig. 2. Modeling memory content loss due to retroactive interference.

In case of cue-dependent forgetting, the learning takes place in combination with the cue. Thus, the association is also linked to a cue (see Fig. 3). If the cue is then not present (dashed line), two scenario are possible: the knowledge object can still be retrieved, due to generalization or the information is lost without the cue, similar to decay.

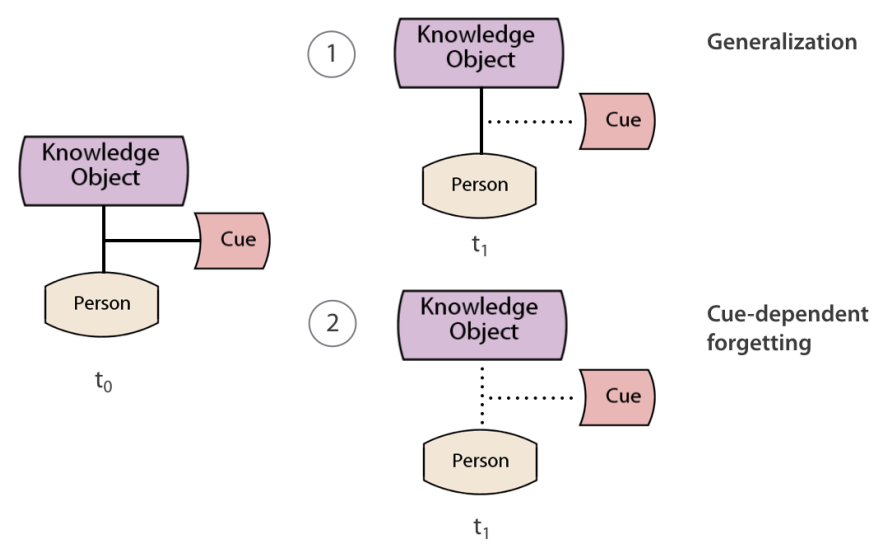

Fig. 3. Modeling memory content loss due to an associated cue missing.

The special case of cue-dependent forgetting, cue-overload, results in even more possible scenarios: when two knowledge objects get associated with one cue, one of the knowledge objects can get lost, both or none (see Fig. 4). This depends on the strength of the association and how much it generalized from the cue. 


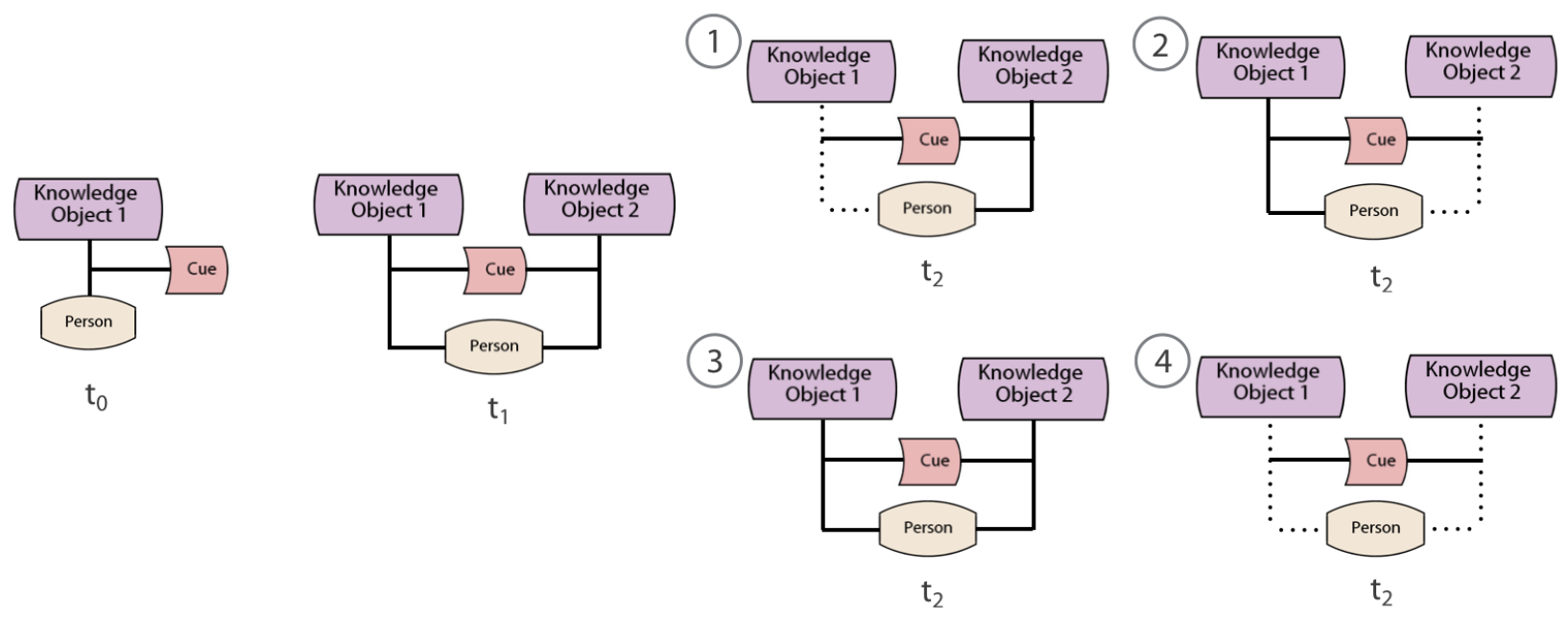

Fig. 4. Modeling memory content loss due to an associated cue being associated with another knowledge object.

In case of intentional forgetting, we cannot predict what happens with the knowledge representation. A not-usage of information could be modeled according to trace-decay. However, it is also possible and for some kind of memory content even more plausible, that unused information cannot be remembered over time due to interference [16].

\section{Demonstration using experimental data}

In order to test the presented modeling approach for forgetting, and especially to better understand the case of intentional forgetting, we provide an example using actual behavioral data from an experiment. In the experiment she learned a special part of the production of (virtual) knee implants. The experiment took place at a digital 4.0 industry research laboratory [20], where the participants learned in teams of three to produce knee implants with the help of an assembly line, diverse machines, robots and tools (in detail, see [14]).

The participants learn the production process according to their working station, thus they acquire special and quite detailed knowledge about the production process. During a first session, participants first learn their part in the production process and then together produce as many knee implants as fast as possible. Within the following three weeks, the participants practice the production routine using an online application at home. At the start of the second session all participants are told that the production process has slightly changed, and that those changes need to be learned and performed. Thus, an instruction to forget parts of the old process is implicitly made. This design allows for the study of intentional forgetting, because we assume that the process learned at the first session has to be intentionally suppressed in order to correctly perform the new process at the second session.

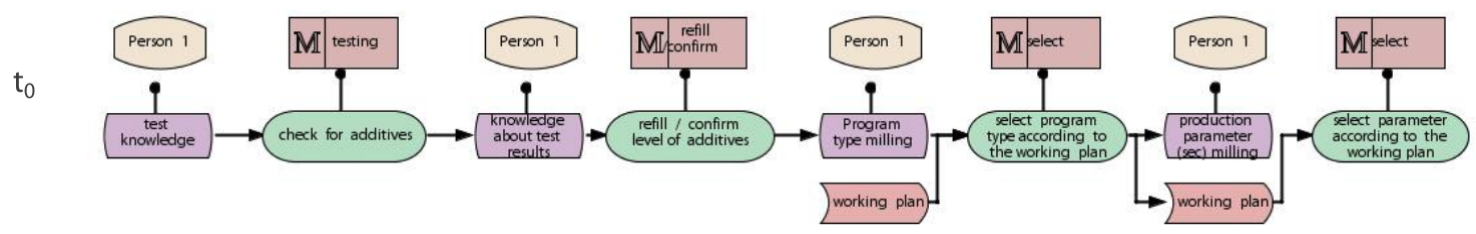

Fig. 5. Modeling a segment of the original production process during the first session.

We demonstrate our modeling approach by focusing on the performance of one participant and her performance for a part of the experiment. The participant was randomly chosen from a pool of participants who performed $100 \%$ correct in the first session according to the instructions. Thus, we assume a stable acquisition of knowledge. We focus on the part of the process (Fig. 5), where 


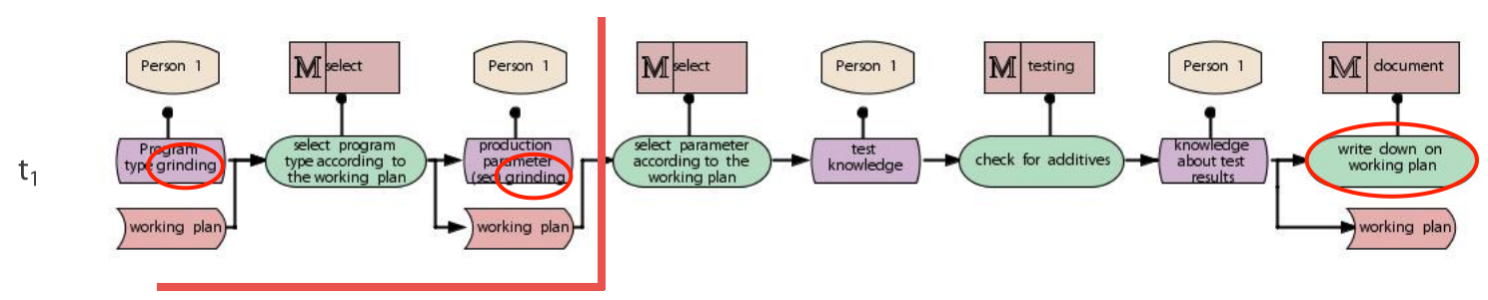

Fig. 6. Modeling a segment of the changes production process during the second session.

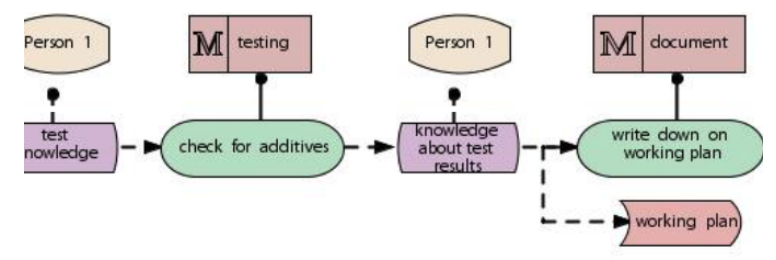

Fig. 7. Modeling the forgotten elements of the newly learned changed production process at the second work piece.

the participant uses her knowledge to test for additives, which the machine needs to work on the blank, and then decides based on the test results whether to refill or simply confirm the level of additives. Further, the participant sets the program type and duration for the milling by checking the working plan and selecting the right parameters on the machine. This is the correct procedure which she over-learned during the first session as well as by using the app at home.

At the second session, a few minor changes are introduced (Fig. 6): instead of milling, only the grinding has to be set for the machine manually, which now has to be done before the additives are checked. Plus, after this testing, the amount of additives have to be written down into the working plan. Thus we have now three changes compared to the original process, which needed to be intentionally forgotten.

At the second session, the participant was first introduced to the changed process by working on one work piece with instructions. After that, the participant worked on four more pieces without the instruction. Observing how she managed to intentionally forget during those four work pieces, it was found that the first of the four work pieces was done correctly, as depicted in (Fig. 6). It is plausible that the instruction and the precise changes are still fresh in mind. The second work piece showed a first deviation from the instruction. She missed to write down the level of additives (Fig. 7). Thus this task is now linked by a dashed line. Work piece three and four both show the same mistakes. Here she fell back into the old routine from the first session and again first checked for additives, actually refilled those and then prepared the machine for grinding. Thus she did not manage to suppress those earlier learned routines and forgot about two of the three changes (Fig. 8).

\section{Outlook: Capturing Dynamic Processes in Business Models}

In order to apply forgetting theories usefully in process business modeling, its tools need to be expanded. A sole focus on the individual level in modeling as demonstrated above is rarely of practical usage. It can be helpful for precise analyses of knowledge development within a certain individual over time. However, interesting implications can be drawn when modeling is implemented for the team and organizational level. Focusing on a group of individuals allows for the analysis of patterns and general working mechanisms. In detail, certain elements which need to be forgotten in order to adapt to a new process can be defined. Thus a change management could include not only the acquisition of knew knowledge which is needed for the future process, but also the elimination of old parts. This could for example be realized by taking away cues which are a good reminder of the obsolete information.

Besides the values for change management, modeling forgetting on a team-level can also be helpful to draw conclusions about the realization of ongoing business processes. In detail, it could be analyzed which parts of a process are easier forgotten than others, taking performance indicators from individual level and aggregate those to analyze the mean performances. To realize this 


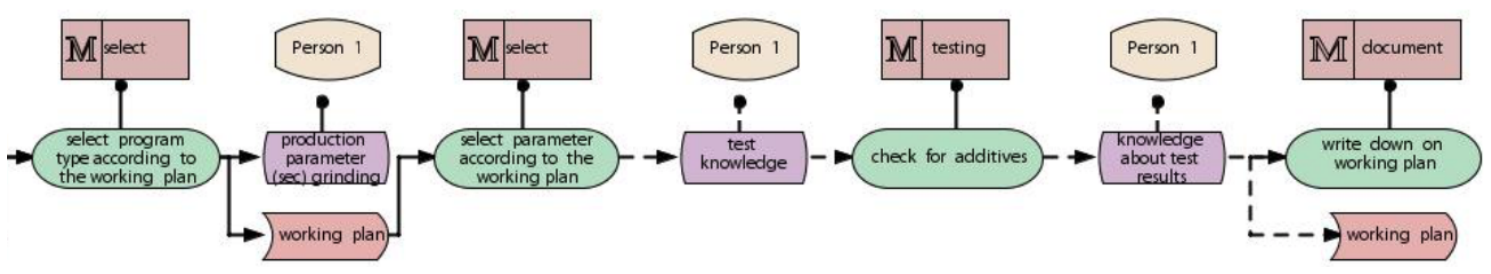

Fig. 8. Modeling the forgotten elements of the newly learned changed production process at the third and fourth work piece.

analyses, modeling needs to track forgotten elements within business processes instead of merely deleting those items. Plus, a representation of time-development beyond the mere comparison of fixed time points is needed in order to address the performance over time, which is crucial for understanding forgetting.

Addressing KMDL, the demands concerning forgetting are threefold: first, enhancing the perspective on knowledge flow to track the forgotten elements throughout an existing process and second the modeling of to be forgotten elements within a business process, so forgetting becomes a standard part of the process. Thus, the modeling would allow for tracking, analysis and planning of forgotten elements, over the past, the status quo and future processes. Third, the possibility to include aspects of the environment into the modeling is needed in order to search for cues or analyze which production environment increases the chance of forgetting. This extension to include environmental factors is not only associated with forgetting processes, but can also be very useful to detect learning- and performance enhancing working mechanism. However, the theory of cue-dependent forgetting makes this especially interesting for the forgetting context.

Although this paper derived KMDL as a suitable language to expand it with the ability to model forgetting, the overall approach could be questioned. As there are no plausible solutions to include forgotten (or to be forgotten) elements into the general process view (as an expansion to this proposed modeling of an individuals performance), maybe a fundamental different approach is needed to account for forgetting in modeling. Especially as the requirements of a clear, comparable and systematical design are not yet met. One way could be by including probabilities along with certain actions or allow for a flexible view of different points in time where missing actions (as to be forgotten actions) could be tracked. The proposed way to model forgetting using KMDL in its current form and to extent it with minimal new features should be seen as a kick-of discussion of how forgetting could be included in modeling approaches.

\section{Conclusion and implications for further research}

For an holistic approach towards knowledge transfer within business processes, the aspect of forgetting needs to be included. As an ordinary and usually beneficial cognitive process, it is a natural part of all human-including work processes. Further, using it as a tool by establishing intentionally to-be-forgotten elements, business processes can be made more efficient [19]. Modeling is a practical method to track, analyze and optimize business processes. First steps to include forgetting into modeling are made here, so individual knowledge development can be tracked. Concerning the different forms of forgetting, environmental influences can be addressed as well, although limited so far. The conduct of parsimony within modeling needs to be stretched in order to further include forgotten elements within business processes for the sake of tracking and analyzing patterns of such. Especially for the team and organizational level more data is needed to test modeling approaches including forgetting features. It can be helpful that also other modeling languages are developed in this direction, thus further theoretical and practical implications can be drawn.

\section{References}

1. Akgün, A.E., Keskin, H., Byrne, J.C., Lynn, G.S.: Organizational unlearning as changes in beliefs and routines in organizations. Journal of Organizational Change Management 20(6), 794-812 (Oct 2007). https://doi.org/10.1108/09534810710831028, https://www .emeraldinsight.com/doi/full/10.1108/ 09534810710831028 
2. Allweyer, T., Scheer, A.: Wissensmanagement mit ARIS-Modellen. Scheer: ARIS-Vom Geschäftsprozess zum Anwendungssystem, Springer (1998)

3. Becker, J., Schütte, R., Geib, T., Ibershoff, H.: Grundsätze ordnungsmäßiger Modellierung (GoM): Sachbericht. Münster: Institut für Wirtschaftsinformatik, Westfälische Wilhelms-Universität (2000)

4. Becker, M.C.: Organizational routines: a review of the literature. Industrial and corporate change 13(4), 643-678 (2004)

5. Bjork, E.L., Bjork, R.A.: Intentional forgetting can increase, not decrease, residual influences of to-beforgotten information. Journal of Experimental Psychology: Learning, Memory, and Cognition 29(4), 524-531 (2003). https://doi.org/10.1037/0278-7393.29.4.524

6. Crowder, R.G.: The interference theory of forgetting in long-term memory. In: Principles of Learning and Memory, pp. 234-279. Psychology Press (2014)

7. Davenport, E., Cronin, B.: Knowledge management: semantic drift or conceptual shift? Journal of Education for library and information Science pp. 294-306 (2000)

8. Dixon, N.M.: The Organizational Learning Cycle : How We Can Learn Collectively. Routledge (Jul 2017). https://doi.org/10.4324/9781315554945

9. França, J.B., Baião, F.A., Santoro, F.M.: Towards characterizing knowledge intensive processes. In: Proceedings of the 2012 IEEE 16th International Conference on Computer Supported Cooperative Work in Design (CSCWD). pp. 497-504. IEEE (2012)

10. Gronau, N.: Wissen prozessorientiert managen: Methode und Werkzeuge für die Nutzung des Wettbewerbsfaktors Wissen in Unternehmen. Oldenburg (2009)

11. Gronau, N., Müller, C., Korf, R.: KMDL-Capturing, Analysing and Improving Knowledge-Intensive Business Processes. J. UCS 11(4), 452-472 (2005)

12. Gronau, N., Thim, C., Ullrich, A., Vladova, G., Weber, E.: A Proposal to Model Knowledge in Knowledge-Intensive Business Processes. BMSD.-Rhodes, Greece 16, 98-103 (2016)

13. Gronau, N., Weber, E.: Management of knowledge intensive business processes. In: International Conference on Business Process Management. pp. 163-178. Springer (2004)

14. Haase, J., Schüffler, A.: Studying cue-dependent and intentional forgetting in organizations: a methodological approach. Prepared for the Hawaii International Conference on System Sciences (HICSS). (2018)

15. Hailikari, T., Katajavuori, N., Lindblom-Ylanne, S.: The Relevance of Prior Knowledge in Learning and Instructional Design. American Journal of Pharmaceutical Education p. 8 (2008)

16. Hardt, O., Nader, K., Nadel, L.: Decay happens: the role of active forgetting in memory. Trends in cognitive sciences 17(3), 111-120 (2013)

17. Heisig, P.: GPO-WM®: Methode und Werkzeuge zum geschäftsprozessorientierten Wissensmanagement. In: Geschäftsprozessorientiertes Wissensmanagement, pp. 47-64. Springer (2002)

18. Hinkelmann, K., Karagiannis, D., Telesko, R.: PROMOTE-Methodologie und Werkzeug für geschäftsprozessorientiertes Wissensmanagement. In: Geschäftsprozessorientiertes Wissensmanagement, pp. 65-90. Springer (2002)

19. Kluge, A., Gronau, N.: Intentional Forgetting in Organizations: The Importance of Eliminating Retrieval Cues for Implementing New Routines. Frontiers in Psychology 9 (2018). https://doi.org/10.3389/fpsyg.2018.00051

20. Lass, S., Gronau, N.: Efficient Analysis of Production Processes with a Hybrid Simulation Environment. In: Proceeding of the 22nd International Conference of Flexible Automation and Intelligent Manufacturing (FAIM 2012), Helsinki, Finnland (2012)

21. Müsseler, J., Rieger, M.: Allgemeine Psychologie. Springer (2002)

22. Netto, J.M., Franca, J.B., Baião, F.A., Santoro, F.M.: A notation for knowledge-intensive processes. In: Computer Supported Cooperative Work in Design (CSCWD), 2013 IEEE 17th International Conference on. pp. 190-195. IEEE (2013)

23. Nonaka, I., Takeuchi, H.: The knowledge creation company: how Japanese companies create the dynamics of innovation. New York: Oxford University Press (1995)

24. Overhage, S., Birkmeier, D.Q., Schlauderer, S.: Qualitätsmerkmale, -metriken und messverfahren für Geschäftsprozessmodelle. Wirtschaftsinformatik 54(5), 217-235 (Oct 2012). https://doi.org/10.1007/s11576-012-0335-1

25. Popov, V., Marevic, I., Rummel, J., Reder, L.: Forgetting is a Feature, not a Bug: Intentionally Forgetting Some Things Helps Us Remember Others by Freeing up Working Memory Resources. OpenPopov, Vencislav et al."Forgetting Is a Feature, Not a Bug: Intentionally Forgetting Some Things Helps Us Remember Others by Freeing up Working Memory Resources". PsyArXiv 6 (2018)

26. Remus, U.: Prozessorientiertes Wissensmanagement. Konzepte und Modellierung. PhD Thesis, Regensburg (2002)

27. Roedinger, H., Weinstein, Y., Agarwal, P.K.: Forgetting: preliminary considerations. In: Forgetting, pp. 15-36. Psychology Press (2010)

28. Rychkova, I., Nurcan, S.: Towards adaptability and control for knowledge-intensive business processes: Declarative configurable process specifications. In: System Sciences (HICSS), 2011 44th Hawaii International Conference on. pp. 1-10. IEEE (2011) 
29. Sadeh, T., Ozubko, J.D., Winocur, G., Moscovitch, M.: Forgetting Patterns Differentiate Between Two Forms of Memory Representation. Psychological Science 27(6), 810-820 (Jun 2016). https://doi.org/10.1177/0956797616638307

30. Schooler, L.J., Hertwig, R.: How forgetting aids heuristic inference. Psychological Review 112(3), 610 (2005)

31. Sigmanek, C., Lantow, B.: A Survey on Modelling Knowledge-intensive Business Processes from the Perspective of Knowledge Management. In: KMIS. pp. 325-332 (2015)

32. Tulving, E.: Cue-dependent forgetting: When we forget something we once knew, it does not necessarily mean that the memory trace has been lost; it may only be inaccessible. American Scientist 62(1), 74-82 (1974)

33. Walsh, J.P., Ungson, G.R.: Organizational memory. Academy of management review 16(1), 57-91 (1991) 